\title{
Effective hard-sphere model of diffusion in aqueous polymer solutions
}

\author{
Stephen S. L. Peppin
}

\begin{abstract}
An effective hard-sphere model of the diffusion and cross-diffusion of salt in unentangled polymer solutions is developed. Given the viscosity, sedimentation coefficient and osmotic pressure of the polymer, the model predicts the diffusion and cross-diffusion coefficients as functions of the polymer concentration and molecular weight. The results are compared with experimental data on $\mathrm{NaCl}$ diffusion in aqueous polyethylene glycol solutions, showing good agreement at polymer molecular weights up to $400 \mathrm{~g} / \mathrm{L}$. At higher molecular weights the model becomes less accurate, likely because of the effects of entanglement. The tracer Fickian diffusivity can be written in the form of a Stokes-Einstein equation containing the solution viscosity. For $\mathrm{NaCl}$ diffusion in polyethylene glycol solutions, the Stokes-Einstein equation breaks down as the polymer size increases. Using Batchelor's viscous correction factor to determine an effective viscosity experienced by the salt ions within the polymer matrix leads to much closer agreement with experiment.
\end{abstract}

\section{Introduction}

The diffusion of a dissolved solute in water can be modelled by Fick's law, with a single diffusion coefficient $D$ quantifying the solute flux in response to a concentration gradient [1]. In aqueous polymer solutions the diffusion is more complex, involving cross-diffusion effects between the solute and polymer, and requiring knowledge of a diffusion coefficient matrix for a complete description [1-3]. The diffusion coefficient matrix of salt in polymer solutions has been measured for several systems $[4,5]$, showing that at high polymer concentrations cross-diffusion effects are significant; in some cases the cross-diffusion coefficients are larger in magnitude than the main Fickian coefficients [6]. In this work a model of cross-diffusion in unentangled, uncharged polymer solutions is obtained by treating the polymers as effective hard spheres. The model uses measurements of the viscosity, osmotic pressure, and sedimentation coefficient of the polymer; given these data explicit expressions for the four diffusion coefficients are obtained as functions of the polymer concentration and molecular weight.

In Section 2 a brief description of cross diffusion in ternary solutions is given; Section 3 reviews Batchelor's hard-sphere cross-diffusion model, and compares the predictions to experimental data on salt diffusion within an aqueous polyethylene glycol (PEG) solution. In Section 4 an effective hard-sphere model is developed, that takes account of departures of the polymer molecules from hard-sphere behaviour. Effective hard-sphere radii and viscous mobilities are defined using the osmotic pressure and viscosity of the binary polymer solutions, leading to improved agreement with experiment. Section 5 extends the model to non-dilute polymer concentrations and contains a discussion of the breakdown of the Stokes-Einstein equation for $\mathrm{NaCl}$ diffusion in PEG solutions.

\section{Cross-diffusion in ternary solutions}

The flux equations describing cross-diffusion in ternary solutions can be written in the form

$$
\boldsymbol{J}_{1}=-D_{11} \nabla n_{1}-D_{12} \nabla n_{2}
$$

For God so loved the world, that He gave His only begotten Son, that whosoever believeth in Him should not perish, but have everlasting life. John 3:16 

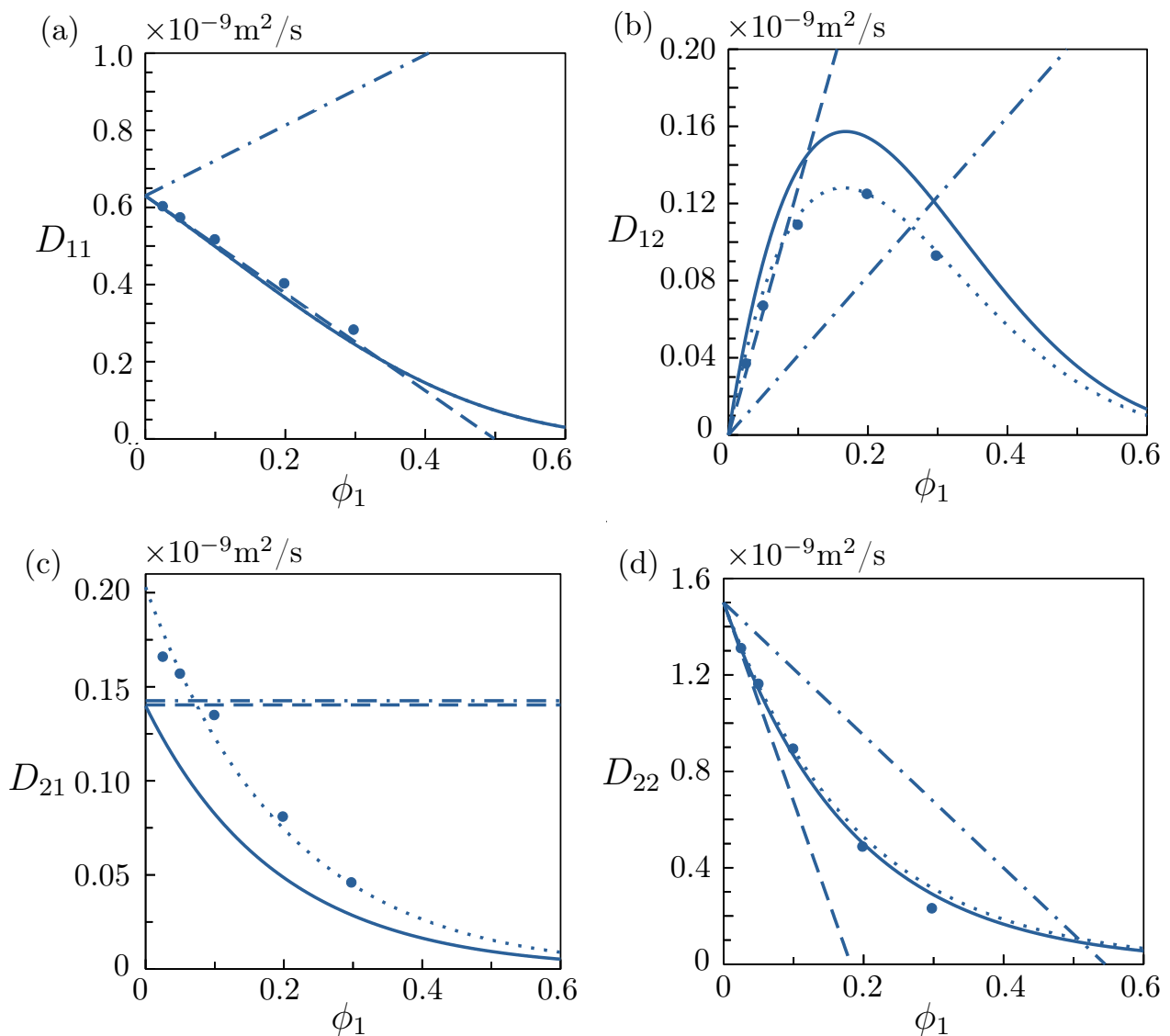

Figure 1: Cross-diffusion coefficients $D_{i j}$ in the ternary solution PEG 200- $\mathrm{NaCl}-\mathrm{H}_{2} \mathrm{O}$ as functions of the PEG hydrodynamic volume fraction $\phi_{1}$. The $\mathrm{NaCl}$ concentration is $0.5 \mathrm{M}\left(\phi_{2}=0.0042\right)$ and the particle size ratio is $\lambda=R_{2} / R_{1}=0.44$. The data (circles) are from Vergara et al. [4]. The dash-dot lines are from the hard-sphere model (10); the dashed lines are from the effective hard-sphere model (19); the solid curves are from the non-dilute effective hard-sphere model (35). The dotted curves are from (35) using the best-fit parameter $\lambda^{\text {fit }}=0.37$.

$$
\boldsymbol{J}_{2}=-D_{21} \boldsymbol{\nabla} n_{1}-D_{22} \boldsymbol{\nabla} n_{2},
$$

where $\boldsymbol{J}_{1}=n_{1}\left(\boldsymbol{v}_{1}-\boldsymbol{v}\right)$ is the flux of the host polymer at concentration $n_{1}$ (number of polymer molecules per unit volume of mixture) moving at speed $\boldsymbol{v}_{1}$ relative to the volume velocity $\boldsymbol{v}$ of the mixture, and $\boldsymbol{J}_{2}=n_{2}\left(\boldsymbol{v}_{2}-\boldsymbol{v}\right)$ is the flux of salt at concentration $n_{2}$. Here $D_{11}$ is the main Fickian diffusion coefficient of the polymer, while $D_{12}$ is a cross-diffusion coefficient characterizing motion of the host molecules caused by a gradient in salt concentration (diffusiophoresis); similarly, $D_{22}$ is the Fickian salt diffusivity, while $D_{21}$ accounts for motion of salt caused by a gradient in the polymer concentration (osmotic diffusion) [7].

Figure 1 shows $D_{i j}$ data (circles) obtained by Vergara et al. [4] for the diffusion of $\mathrm{NaCl}$ within a matrix of polyethylene glycol (PEG) molecules in water. PEG is a hydrophilic, uncharged polymer with many uses in the food, medicine and biotechnology industries, and can be prepared in a range of molecular weights [4]. PEG interacts with other particles in water mainly via excluded volume effects, and is therefore a relatively good system to apply a hard-sphere diffusion model [6]. However, as will be seen in Sections 3.2 and 4.1.2, PEG exhibits significant departures from hard-sphere behaviour even in the dilute limit.

The maximum PEG concentration in the experiments was $C_{1}=3.0 \mathrm{M}=3000 \mathrm{~mol} / \mathrm{m}^{3}$, corresponding to a hydrodynamic volume fraction $\phi_{1}=n_{1} v_{1}=0.30$, where $n_{1}=N a C_{1}$ is the PEG number density, $N a$ is Avogadro's number, $v_{1}=\frac{4}{3} \pi R_{1}^{3}$ is the hydrodynamic volume of a PEG 
molecule and $R_{1}=0.34 \times 10^{-9} \mathrm{~m}$ is the PEG hydrodynamic radius [8]. Because of the relatively small globular size of the PEG molecules (molecular weight 194 [4]), the polymer molecules were not significantly entangled [9]; however, at these high volume fractions the solution is crowded and the $D_{i j}$ data is nonlinear, with the polymer cross-diffusion coefficient $D_{12}$ showing a maximum near $\phi_{1}=0.2$.

The concentration of $\mathrm{NaCl}$ in the diffusion experiments was $0.5 \mathrm{M}$, corresponding to a hydrodynamic volume fraction $\phi_{2}=n_{2} v_{2}=0.0042$, where $n_{2}=N a C_{2}$ is the NaCl number density, $v_{2}=\frac{4}{3} \pi R_{2}^{3}$ and $R_{2}=0.15 \mathrm{~nm}$ is the $\mathrm{NaCl}$ hydrodynamic radius [10]. The $\mathrm{NaCl}$ main Fickian diffusion coefficient $D_{22}$ in figure 1d decreases rapidly as the PEG concentration increases, reflecting the obstruction effect of the PEG molecules on the diffusion of salt. The relatively large measured values of the cross coefficients $D_{12}$ and $D_{21}$ indicate the importance of cross-diffusion on the motion of $\mathrm{NaCl}$ in this system [4].

\section{Batchelor's cross-diffusion model}

In this section Batchelor's [3] theory of tracer cross-diffusion in hard-sphere suspensions is briefly reviewed and applied to the PEG diffusion data. From nonequilibrium thermodynamics [11, 12] the four diffusion coefficients in a ternary solution can be written in the form

$$
D_{i j}=\sum_{k=1}^{2} L_{i k} \mu_{k j} \quad(i, j=1,2),
$$

where $L_{i j}=L_{j i}$ are Onsager phenomenological coefficients, $\mu_{i}$ are the reduced chemical potentials per particle and $\mu_{i j}=\left(\partial \mu_{i} / \partial n_{j}\right)_{T, P, n_{k \neq j}}=\mu_{j i}[3,11-13]$. In the following the temperature $T$ and mixture pressure $P$ are assumed constant. In the McMillan-Mayer theory of suspensions the reduced chemical potentials are equal to the chemical potentials of a binary hard-sphere gas, and their derivatives can be written in the dilute limit as

$$
\mu_{i j}=k_{B} T\left(\frac{\delta_{i j}}{n_{i}}+2 B_{i j}\right) \quad(i, j=1,2),
$$

where $k_{B}$ is Boltzmann's constant, $\delta_{i j}$ is the delta function and $B_{i j}$ are the osmotic virial coefficients accounting for interparticle forces [12, 14]. To first order in the volume fractions $\phi_{i}=v_{i} n_{i}$, the phenomenological coefficients $L_{i j}$ in a hard-sphere suspension are

$$
L_{i j}=\frac{n_{i}}{6 \pi \eta_{0} R_{i}}\left[\delta_{i j}\left(1+\sum_{k=1}^{2} K_{i k}^{\prime} \phi_{k}\right)+\lambda_{i j}^{-3} K_{i j}^{\prime \prime} \phi_{j}\right] \quad(i, j=1,2),
$$

where $\eta_{0}$ is the solvent viscosity and $K_{i j}^{\prime}$ and $K_{i j}^{\prime \prime}$ are mobility coefficients accounting for viscous interactions between the particles; for hard spheres the mobility coefficients are functions of the particle size ratio $\lambda_{i j}=R_{j} / R_{i}[3,13]$. In general the mobility coefficients $K_{i j}^{\prime}$ and $K_{i j}^{\prime \prime}$ obey the relations

$$
\lim _{\lambda_{21} \rightarrow 0} K_{21}^{\prime}=-k_{\eta}, \quad K_{11}^{\prime}+K_{11}^{\prime \prime}=-k_{s} \quad \text { and } \quad \frac{K_{12}^{\prime \prime}}{\lambda_{12}}=\frac{K_{21}^{\prime \prime}}{\lambda_{21}},
$$

where $k_{\eta}$ is the viscosity concentration coefficient and $k_{s}$ is the sedimentation concentration coefficient, described in more detail in Section 4.1. For hard spheres $k_{\eta}=2.5$ and $k_{s}=6.55$ [3]. Equation $(5 a)$ ensures that when the tracer particles are much larger than the host particles, the effect of the host matrix is to increase the effective viscosity of the fluid by the factor $1+k_{\eta} \phi_{1}$. Similarly, $(5 b)$ ensures that when the particles are all the same size $\left(\lambda_{i j}=1\right)$, the sedimentation coefficient of the particles is the same as for a monodisperse system with total volume fraction $\phi=\phi_{1}+\phi_{2}$, and is smaller than the sedimentation coefficient of a single particle by the factor $1-k_{s} \phi[3]$. Equation $(5 c)$ is a consequence of the Onsager reciprocal relation $L_{12}=L_{21}$ [13].

Combining equations (2), (3) and (4) in the dilute tracer limit $\phi_{2} \ll \phi_{1} \ll 1$ gives, upon neglecting terms of order $\phi_{i}^{2}$,

$$
D_{11}=D_{1}^{0}\left(1+\left[b_{11}+K_{11}^{\prime}+K_{11}^{\prime \prime}\right] \phi_{1}\right), \quad D_{12}=D_{1}^{0}\left(b_{12}+K_{12}^{\prime \prime}\right) \phi_{1},
$$




$$
D_{21}=D_{2}^{0}\left(b_{21}+K_{21}^{\prime \prime}\right) \phi_{2}, \quad D_{22}=D_{2}^{0}\left(1+K_{21}^{\prime} \phi_{1}\right),
$$

where $D_{i}^{0}=k_{B} T /\left(6 \pi R_{i} \eta_{0}\right)$ is the Stokes-Einstein diffusivity of component $i$ in the pure solvent and $b_{i j}=2 B_{i j} / v_{i}[3,15]$.

\subsection{Hard-sphere virial coefficients and viscous mobilities}

For hard-sphere particles the virial coefficients depend only on the particle radii and are given by the expression [12]

$$
B_{i j}=\frac{2}{3} \pi\left(R_{i}+R_{j}\right)^{3}
$$

so that

$$
b_{i j}=\frac{2 B_{i j}}{v_{i}}=\left(1+\lambda_{i j}\right)^{3} .
$$

Batchelor [3] obtained the following approximate expressions for the mobility coefficients of hard-sphere suspensions ${ }^{1}$

$$
K_{i j}^{\prime}=-\frac{2.5}{\left(1+b \lambda_{i j}\right)} \quad \text { and } \quad K_{i j}^{\prime \prime}=\frac{\lambda_{i j}^{2}}{\left(1+\lambda_{i j}^{3}\right)}-\left(\lambda_{i j}^{2}+3 \lambda_{i j}+1\right)
$$

where $b$ is a numerical coefficient that in general depends on the $\lambda_{i j}$. In the case $\lambda_{i j}=1, b=0.22$ is required to match equation $(5 b)$ for hard spheres $[15,16]$. In the Corrigendum to [3], Batchelor showed that using the value $b=0.16$ allows for agreement of $(9 a)$ with the results of numerical simulations (to within $5 \%$ ) at all $\lambda_{i j}$, and his value for $b$ is used in the following.

\section{$3.2 \quad$ Hard-sphere results}

With (8) and (9), equations (6) can be written in the form

$$
\begin{gathered}
D_{11}=D_{1}^{0}\left(1+1.45 \phi_{1}\right), \quad D_{12}=D_{1}^{0}\left(\lambda^{3}+2 \lambda^{2}+\frac{\lambda^{2}}{1+\lambda^{3}}\right) \phi_{1}, \\
D_{21}=D_{2}^{0}\left(\lambda^{-3}+2 \lambda^{-2}+\frac{\lambda^{-2}}{1+\lambda^{-3}}\right) \phi_{2}, \quad D_{22}=D_{2}^{0}\left(1-\frac{2.5}{1+0.16 / \lambda} \phi_{1}\right),
\end{gathered}
$$

where $\lambda=\lambda_{12}=R_{2} / R_{1}$. Actually, inserting (8) and (9) into (6a) gives $D_{11}=D_{1}^{0}\left(1+1.34 \phi_{1}\right)$, rather than the more exact result $(10 a)$. The relatively small difference is caused by the value of $b$ used in equation $(9 a)[3,15]$.

The Stokes-Einstein diffusivity of PEG 200 in water at $25^{\circ} \mathrm{C}$ is $D_{1}^{0}=0.63 \times 10^{-9} \mathrm{~m}^{2} / \mathrm{s}$, and the hydrodynamic radius is $R_{1}=k_{B} T /\left(6 \pi \eta_{0} D_{1}^{0}\right)=0.34 \mathrm{~nm}$ [4]. For $\mathrm{NaCl}$ the Stokes-Einstein

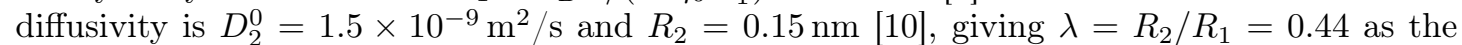
particle size ratio. The dash-dot lines in figure 1 show the diffusion coefficients calculated from (10) with $\lambda=0.44$. The hard-sphere model does not agree very well with the $D_{i j}$ data, suggesting that although PEG molecules are relatively globular and inert, they do not behave like hard spheres in aqueous solution. In the next section this is confirmed using osmotic pressure and viscosity data, and a more general model is developed that takes into account the unique hydrodynamic and thermodynamic properties of the PEG molecules.

\footnotetext{
${ }^{1}$ Batchelor [13] proved that in general for low Peclet number flows the $K_{i j}^{\prime \prime}$ obey the Onsager relation $(5 c)$. The approximate equations (9), however, do not exactly satisfy the Onsager relation nor the requirement $(5 b)$ when $\lambda_{i j}=1$. The slightly modified equations

$$
K_{i j}^{\prime}=-\frac{2.5}{\left(1+b \lambda_{i j}\right)} \quad \text { and } \quad K_{i j}^{\prime \prime}=\frac{\lambda_{i j}^{2.5}}{\left(1+\lambda_{i j}^{3}\right)}-\left(\lambda_{i j}^{2}+3 \lambda_{i j}+1\right)
$$

with $b=0.22$ agree closely with (9) for $\lambda_{i j}=1$ (to within $5 \%$ ) while also satisfying the conditions in (5) at all $\lambda_{i j}$. As the differences are relatively small Batchelor's equations (9) are used in the remainder of this work.
} 


\section{Effective hard-sphere model}

In many polymer and colloidal solutions an effective hard-sphere radius $R_{1}^{e}$ can be used in place of the hydrodynamic radius $R_{1}$, leading to improved agreement between experiment and hard-sphere models [17-19]. In this section a similar effective hard-sphere approach is developed in which osmotic pressure and viscosity data are used to define an effective particle radius and effective viscous mobility coefficients. In order to properly define these quantities a brief discussion of viscosity, diffusion, sedimentation and osmotic pressure in polymer solutions is given below.

\subsection{Hydrodynamic coefficients in polymer solutions}

To first order in concentration the viscosity $\eta$, mutual diffusion coefficient $D$, sedimentation coefficient $s$ and osmotic pressure $\Pi$ of a binary polymer-solvent solution can be written as

$$
\begin{array}{r}
\eta=\eta_{0}\left(1+k_{\eta} \phi_{1}\right), \quad D=D_{0}\left(1+k_{D} \phi_{1}\right), \\
s=s_{0}\left(1-k_{s} \phi_{1}\right), \quad \Pi=\Pi_{0}\left(1+k_{\Pi} \phi_{1}\right) .
\end{array}
$$

In $(11 a) \eta_{0}$ is the solvent viscosity and $k_{\eta}$ is the viscosity concentration coefficient; in $(11 b)$ $D_{0}=k_{B} T /\left(6 \pi R_{1} \eta_{0}\right)$ is the Stokes-Einstein diffusivity of a polymer molecule in the pure fluid solvent and $k_{D}$ is the diffusion concentration coefficient. Similarly, in $(11 c) s_{0}$ is the dilute-limit polymer sedimentation coefficient and $k_{s}$ is the sedimentation concentration coefficient; and in (11d) $\Pi_{0}=n_{1} k_{B} T$ is the dilute-limit polymer osmotic pressure and $k_{\Pi}=B_{11} / v_{1}=b_{11} / 2$ is the osmotic concentration coefficient, where $B_{11}$ is the second virial coefficient [16, 20, 21]. For hard-sphere particles the concentration coefficients in (11) have the known values

$$
k_{\eta}=2.5, \quad k_{\Pi}=4, \quad k_{s}=6.55 \quad \text { and } \quad k_{D}=1.45,
$$

independent of the particle size $[16,21]$. For polymers, the values of $k_{D}, k_{\eta}, k_{\Pi}$ and $k_{s}$ often depart from (12) and typically depend on the molecular weight $M_{1}$ [8].

The concentration coefficients in (11) are not all independent but are related by the equations

$$
k_{D}=2 k_{\Pi}-k_{s} \quad \text { and } \quad k_{s}=\mathcal{R} k_{\eta} .
$$

Equation (13a) is a consequence of the generalized Stokes-Einstein equation [16, 20, 21], while $(13 b)$ is the empirical Wales-van Holde relation, where $\mathcal{R}$ is the Wales-van Holde ratio [20, 22].

\subsubsection{Effective hard-sphere radius}

The polymer effective (osmotic) volume is defined as $v_{1}^{e} \equiv v_{1}\left(k_{\Pi} / 4\right)=B_{11} / 4[18,21]$ so that the effective hard-sphere radius is

$$
R_{1}^{e}=R_{1}\left(\frac{k_{\Pi}}{4}\right)^{1 / 3} .
$$

With equation (14) the effective radius can be determined given the osmotic concentration coefficient $k_{\Pi}$. For true hard-sphere particles $k_{\Pi}=4$ and $R_{1}^{e}=R_{1}$.

\subsubsection{Application to polyethylene glycol}

Table 1 gives the Stokes-Einstein diffusivity $D_{1}^{0}$, hydrodynamic radius $R_{1}$ and concentration coefficients of PEG in water for three molecular weights [8]. The viscosity coefficient $k_{\eta}$ values in Table 1 have been determined from figure $2 a$, which shows measurements of the viscosity $\eta$ of $\mathrm{PEG}-\mathrm{H}_{2} \mathrm{O}$ solutions as a function of $\phi_{1}$. To extract the viscosity concentration coefficient $k_{\eta}$, the data in figure 2 has been fit to the exponential equation [24, 25]

$$
\eta=\eta_{0} \mathrm{e}^{k_{\eta} \phi_{1}}
$$

which reduces to $(11 b)$ in the dilute limit. The PEG viscosity coefficient is significantly larger than the hard-sphere value of 2.5 at all molecular weights. This is a consequence of the hydrated, 

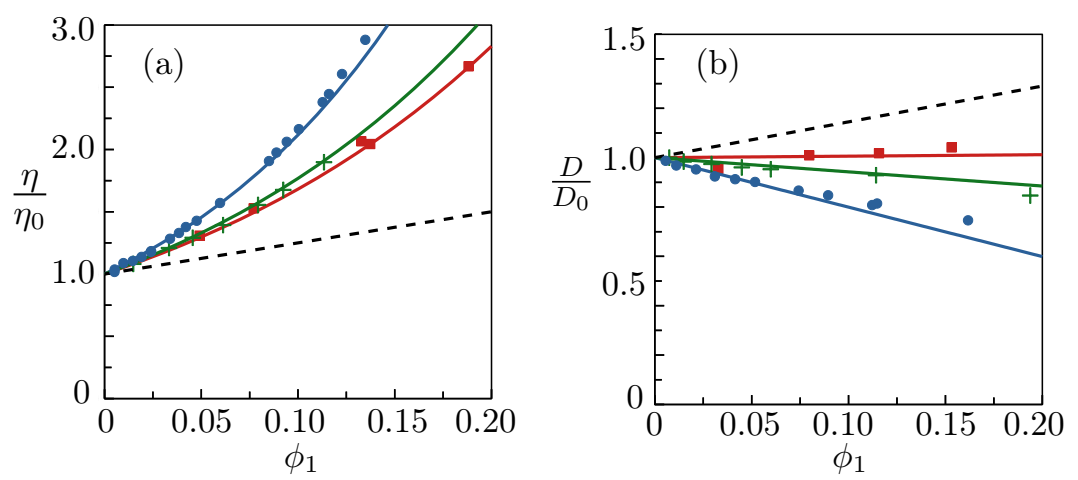

Figure 2: (a) Relative viscosity $\eta\left(\phi_{1}\right) / \eta_{0}$ of PEG- $\mathrm{H}_{2} \mathrm{O}$ solutions measured at $25^{\circ} \mathrm{C}$ by Albright et al. [23] (PEG 200, circles; PEG 2000, squares) and by Vergara et al. [6] (PEG 400, plusses). The curves are from equation (15) giving the viscosity concentration coefficient $k_{\eta}$ values in Table 1. The dashed line is from equation ( $11 a$ ) using the hard-sphere value $k_{\eta}=2.5$. (b) $\mathrm{PEG}-\mathrm{H}_{2} \mathrm{O}$ mutual diffusion coefficient $D\left(\phi_{1}\right)$ measured at $25^{\circ} \mathrm{C}$ by Vergara et al. [8] (PEG 200, circles; PEG 400, crosses; PEG 2000, squares). The solid lines are from equations (11b) and (13a) using the coefficient values in Table 1, while the dashed line is the hard-sphere case with $k_{D}=1.45$.

\begin{tabular}{lccccccccc}
\hline & $D_{1}^{0}\left(\times 10^{-9} \mathrm{~m}^{2} / \mathrm{s}\right)$ & $R_{1}(\mathrm{~nm})$ & $k_{\Pi}$ & $k_{\eta}$ & $k_{s}$ & $k_{D}$ & $R_{1}^{e}(\mathrm{~nm})$ & $\lambda$ & $\lambda^{\text {fit }}$ \\
\hline PEG 200 & 0.63 & 0.34 & 1.81 & 7.5 & 5.6 & -2.0 & 0.26 & 0.44 & 0.37 \\
PEG 400 & 0.44 & 0.49 & 1.85 & 5.7 & 4.3 & -0.6 & 0.38 & 0.31 & 0.25 \\
PEG 2000 & 0.20 & 1.09 & 1.98 & 5.2 & 3.9 & 0.06 & 0.86 & 0.14 & 0.11 \\
\hline
\end{tabular}

Table 1: Physical parameters for PEG- $\mathrm{H}_{2} \mathrm{O}$ solutions at $25^{\circ} \mathrm{C}$.

globular nature of the PEG molecules, which tend to interact with each other more strongly in a shear flow than would occur for true hard-spheres [24]. Note that at a given hydrodynamic volume fraction the PEG 200 viscosity in figure $2 a$ is largest, followed by PEG 400 and PEG 2000. This is because the larger PEG molecules adsorb more water per unit mass increasing their hydrodynamic volumes; when plotted versus the molar concentration $C_{1}$ or mass fraction $w_{1}$ the viscosity of PEG 2000 is largest, followed by PEG 400 and PEG 200 [6, 23].

The osmotic concentration coefficients $k_{\Pi}$ in Table 1 have been obtained from measurements of the second virial coefficient by Yasukawa et al. [26] (Appendix A). The osmotic concentration coefficient in all three cases is smaller than the hard-sphere value $k_{\Pi}=4$. The effective PEG radius can be calculated from equation (14) as $R_{1}^{e}=R_{1}\left(k_{\Pi} / 4\right)^{1 / 3}$, giving the values shown in Table 1 . The relatively large hydrodynamic radius $R_{1}$ reflects the hydrophilic nature of PEG molecules, which tend to adsorb water molecules within the polymer coils and drag the adsorbed water along during motion [9].

The sedimentation concentration coefficient $k_{s}$ has been measured for many polymers [27] but is not yet available for PEG. Given $k_{\eta}$, however, the sedimentation concentration coefficient can be estimated from equation $(13 b)$ as $k_{s}=\mathcal{R} k_{\eta}$. For hard spheres the Wales-van Holde ratio is $\mathcal{R}=6.55 / 2.5=2.62$, while for polymers and macromolecules $\mathcal{R}$ typically varies between 0.2 for rod-like molecules and 1.6 for globular particles [28]. Low molecular weight PEG acts like an ideal polymer [29], in which case $\mathcal{R}=0.75$ [30]; using this value for $\mathcal{R}$ gives the $k_{s}$ values shown in Table 1.

Given $k_{s}$, the diffusion concentration coefficient $k_{D}$ can be obtained from equation $(13 a)$ as $k_{D}=2 k_{\Pi}-k_{s}$. Figure $2 b$ shows $D\left(\phi_{1}\right)$ calculated from equations (11b) and (13) (solid lines), along with experimental PEG- $\mathrm{H}_{2} \mathrm{O}$ mutual diffusion coefficient data from Vergara et al. showing good agreement. [8]. The dashed line in figure $2 b$ shows the hard-sphere case $\left(k_{D}=1.45\right)$; as with the viscosity and osmotic pressure the PEG diffusion coefficient shows significant departures from hard-sphere behaviour, and the difference depends on the polymer molecular weight. The 


\begin{tabular}{cccc}
\hline & $D_{2}^{0}\left(\times 10^{-9} \mathrm{~m}^{2} / \mathrm{s}\right)$ & $R_{2}(\mathrm{~nm})$ & $i$ \\
\hline $\mathrm{NaCl}$ & 1.5 & 0.15 & 1.8 \\
\hline
\end{tabular}

Table 2: Physical parameters for $0.5 \mathrm{M} \mathrm{NaCl}-\mathrm{H}_{2} \mathrm{O}$ solutions at $25^{\circ} \mathrm{C}$.

good agreement between the data in figure $2 b$ and equation (13) suggests that the departure of the PEG diffusion from hard-sphere behaviour is related to the osmotic pressure and viscosity of the polymer.

\subsection{3 $\mathrm{NaCl}$}

The hydrodynamic radius $R_{2}$ of $\mathrm{NaCl}$ at $25^{\circ} \mathrm{C}$ is $0.15 \mathrm{~nm}$ and the Stokes-Einstein diffusivity in water is $D_{2}^{0}=1.5 \times 10^{-9} \mathrm{~m}^{2} / \mathrm{s}[4,10]$ (Table 2). Here it is assumed that the $\mathrm{NaCl}$ osmotic radius is approximately equal to the hydrodynamic radius, $R_{2}^{e} \approx R_{2}$. Also shown in Table 2 is the $\mathrm{NaCl}$ van't Hoff coefficient $i$ evaluated at $C_{2}=0.5 \mathrm{M}$ [31], which will be used in Section 5 .

\subsection{Effective virial coefficients and viscous mobilities}

In the effective hard-sphere model the osmotic virial coefficients $(7)$ become $B_{i j}=\frac{2}{3} \pi\left(R_{i}^{e}+R_{j}^{e}\right)^{3}$ so that

$$
b_{i j}=\frac{2 B_{i j}}{v_{i}}=\left(\frac{R_{i}^{e}+R_{j}^{e}}{R_{i}}\right)^{3}
$$

in place of (8). The hydrodynamic mobilities $K_{i j}^{\prime}$ and $K_{i j}^{\prime \prime}$ will also depart from the hard-sphere expressions (9) because of the asphericity, flexibility and hydration of the polymer molecules [32-34]. Here it is assumed that for relatively globular, unentangled polymers effective mobility coefficients can be defined as

$$
K_{i j}^{\prime}=a^{\prime}\left(\frac{-2.5}{\left(1+0.16 \lambda_{i j}\right)}\right) \quad \text { and } \quad K_{i j}^{\prime \prime}=a^{\prime \prime}\left(\frac{\lambda_{i j}^{2}}{\left(1+\lambda_{i j}^{3}\right)}-\left(\lambda_{i j}^{2}+3 \lambda_{i j}+1\right)\right),
$$

where the viscous factor $a^{\prime}$ and asphericity factor $a^{\prime \prime}$ can be obtained from the PEG viscosity data. Combining $(17 a, b)$ with $(5 a, b)$ gives

$$
a^{\prime}=\frac{k_{\eta}}{2.5} \quad \text { and } \quad a^{\prime \prime}=\left(\frac{k_{s}}{4.5}-\frac{k_{\eta}}{5.5}\right) .
$$

\subsection{Effective hard-sphere diffusivities}

Using (16)-(18) in (6) and assuming $R_{2}^{e} \approx R_{2}$ gives the following effective hard-sphere expressions for the cross-diffusion coefficients, bearing in mind that $\lambda=\lambda_{12}=\lambda_{21}^{-1}, k_{\Pi}=b_{11} / 2$ and $K_{11}^{\prime}+K_{11}^{\prime \prime}=-k_{s}$

$$
\begin{gathered}
D_{11}=D_{1}^{0}\left[1+\left(2 k_{\Pi}-k_{s}\right) \phi_{1}\right] \\
D_{12}=D_{1}^{0}\left[\left(\frac{R_{1}^{e}}{R_{1}}+\lambda\right)^{3}+a^{\prime \prime}\left(\frac{\lambda^{2}}{1+\lambda^{3}}-\left(\lambda^{2}+3 \lambda+1\right)\right)\right] \phi_{1}, \\
D_{21}=D_{2}^{0}\left[\left(1+\frac{R_{1}^{e}}{R_{1}} \lambda^{-1}\right)^{3}+a^{\prime \prime}\left(\frac{\lambda^{-2}}{1+\lambda^{-3}}-\left(\lambda^{-2}+3 \lambda^{-1}+1\right)\right)\right] \phi_{2}, \\
D_{22}=D_{2}^{0}\left(1-\frac{k_{\eta} \phi_{1}}{1+0.16 / \lambda}\right) .
\end{gathered}
$$

Equations (19) are plotted as the dashed lines in figure 1, using the $k_{\Pi}, k_{\eta}$ and $k_{s}$ values given in Table 1, showing better agreement with the experimental data. However, the model does not capture the $\phi_{1}$ dependence of the $\mathrm{NaCl}$ osmotic-diffusion coefficient $D_{21}$, nor the nonlinearities in the data at higher PEG concentrations. To account for these effects in the next section a non-dilute effective hard-sphere model is developed. 


\section{Concentrated solutions}

For tracer diffusion in concentrated solutions the diffusivities $D_{i j}$ can be written in the form

$$
\begin{gathered}
D_{11}=n_{1} \frac{k}{\eta_{0}} \Pi_{1}, \quad D_{12}=n_{1} \frac{k}{\eta_{0}} \sigma \pi_{2}, \\
D_{21}=\frac{n_{2}}{n_{1}} \ell D_{11}-\gamma D_{t}, \quad D_{22}=D_{t},
\end{gathered}
$$

where $k$ is the permeability and $\Pi$ the osmotic pressure of the host-particle matrix, $\pi$ is the osmotic pressure of the tracer particles in the pores of the matrix, $\Pi_{i}=\left(\partial \Pi / \partial n_{i}\right)_{T, P, n_{j}}$ and $\pi_{i}=\left(\partial \pi / \partial n_{i}\right)_{T, P, n_{j}}$ are derivatives of the osmotic pressure with respect to concentration, $\gamma=-\pi_{1} / \pi_{2}$ is the host/tracer preferential interaction coefficient, $\sigma$ is the reflection coefficient of the host matrix, $D_{t}$ is the tracer diffusivity within the pore space, $\ell=(\sigma+\alpha-1) / \alpha$ is a cross-diffusion factor and $\alpha$ is the equilibrium partition coefficient of the tracer between the pore space and bulk solvent [15].

\subsection{Osmotic pressure}

The osmotic pressure of the host-particle matrix can be written as

$$
\Pi=n_{1} k_{B} T Z,
$$

where $Z$ is the compressibility factor (osmotic coefficient). Assuming the $\mathrm{NaCl}$ concentration is sufficiently low that it does not significantly affect the uncharged polymer osmotic pressure (i.e. assuming $\Pi_{2}=0$ ), in the effective hard-sphere model $Z$ is given at volume fractions up to 0.55 by the Carnahan-Starling equation

$$
Z=\frac{1+\phi_{1}^{e}+\phi_{1}^{e 2}-\phi_{1}^{e 3}}{\left(1-\phi_{1}^{e}\right)^{3}},
$$

where $\phi_{1}^{e}=v_{1}^{e} n_{1}=\phi_{1} k_{\Pi} / 4$ is the effective (osmotic) volume fraction and $v_{1}^{e}$ is the PEG effective volume defined in Section 4.1.1 [18,35]. Differentiating (21) with respect to $n_{1}$ gives, bearing in mind that $v_{1}^{e}$ is a constant,

$$
\Pi_{1}=\left(\frac{\partial n_{1} k_{B} T Z}{\partial n_{1}}\right)_{T, P, n_{2}}=k_{B} T\left(\frac{\mathrm{d} \phi_{1}^{e} Z}{\mathrm{~d} \phi_{1}^{e}}\right)=k_{B} T\left[\frac{1+4 \phi_{1}^{e}+4 \phi_{1}^{e 2}-4 \phi_{1}^{e 3}+\phi_{1}^{e 4}}{\left(1-\phi_{1}^{e}\right)^{4}}\right] .
$$

The salt osmotic pressure in the pore fluid is

$$
\pi=i n_{r} k_{B} T,
$$

where $i$ is the van't Hoff factor, $n_{r}=n_{2} / \alpha$ is the salt concentration in the pore space and $\alpha$ is the salt partition coefficient between the pores and the bulk fluid $[15,36]$,

$$
\alpha=\mathrm{e}^{-b_{12} \phi_{1}} .
$$

Here $b_{12}$ is given by equation (16) as $b_{12}=2 B_{12} / v_{1}=\left(R_{1}^{e} / R_{1}+\lambda\right)^{3}$, assuming as in Section 4.3 that $R_{2}^{e}=R_{2}$. For $\mathrm{NaCl}$ in the infinitely dilute limit the $\mathrm{Na}^{+}$and $\mathrm{Cl}^{-}$ions completely dissociate and $i=2$; at higher concentrations $i$ is less than 2 (partial dissociation), and at $C_{2}=0.5 \mathrm{M}$ $i=1.8[31]$.

Differentiating (24) with respect to $n_{i}$ gives

$$
\pi_{1}=i k_{B} T n_{2} b_{12} v_{1} / \alpha \quad \text { and } \quad \pi_{2}=i k_{B} T / \alpha,
$$

from which the preferential interaction coefficient $\gamma$ can be obtained as

$$
\gamma=-\pi_{1} / \pi_{2}=-n_{2} b_{12} v_{1}=-\phi_{2} b_{12} / \lambda^{3} .
$$

Equations (25) and (27) account for the fact that the PEG molecules tend to preferentially adsorb water molecules while rejecting the $\mathrm{Na}^{+}$and $\mathrm{Cl}^{-}$ions into the pore fluid, so that $n_{r}$ in the pore space is larger than the bulk salt concentration $n_{2}[12,36]$. 


\subsection{Permeability}

The permeability of the host matrix to the flow of water can be written as

$$
k=\frac{f}{6 \pi R_{1} n_{1}},
$$

where $f=s / s_{0}$ is the dimensionless sedimentation coefficient (friction coefficient), given by (11c) in the dilute limit $[17,37]$. Here it is assumed that for relatively globular polymer molecules $f$ is given by a hard-sphere Richardson-Zaki equation [17, 38]

$$
f=\left(1-\phi_{1}\right)^{k_{s}},
$$

where $k_{s}$ is the sedimentation concentration coefficient obtained in Section 4.1.2. Equation (29) reduces to equation $(11 c)$ in the limit $\phi_{1} \rightarrow 0$.

\subsection{Tortuosity and reflection coefficient}

The tracer diffusivity $D_{t}$ can be written in the form

$$
D_{t}=\tau D_{2}^{0}
$$

where $\tau$ is the diffusive tortuosity factor accounting for the hindrance effect of the polymer matrix on the tracer particle diffusion $[15,39]$. In the dilute limit $\phi_{1} \rightarrow 0$, comparison of $(30)$ and $(20 d)$ with $(6 d)$ gives

$$
\tau=1-\tau_{1} \phi_{1},
$$

where $\tau_{1}=-K_{21}^{\prime}$. Similarly, equation $(20 b)$ in the limit $\phi_{1} \rightarrow 0$ becomes $D_{12}=D_{1}^{0} \sigma$, and comparison with $(6 b)$ gives

$$
\sigma=\sigma_{1} \phi_{1}
$$

where $\sigma_{1}=b_{12}+K_{12}^{\prime \prime}$.

In the hard sphere case, constitutive equations for $\tau$ and $\sigma$ in concentrated suspensions have been obtained by using capillary flow models and a power-law equation for the hard-sphere viscosity [15]. For unentangled polymer solutions the viscosity is more accurately described by exponential functions [24, 25], as demonstrated for PEG in Section 4.1.2. By analogy with equation (15) this suggests constitutive equations of the form

$$
\tau=\mathrm{e}^{-\tau_{1} \phi_{1}} \quad \text { and } \quad \sigma=1-\mathrm{e}^{-\sigma_{1} \phi_{1}},
$$

which reduce to (31) and (32) in the dilute limit.

The tortuosity $\tau$ and reflection coefficient $\sigma$ measure the degree to which the salt ions can diffuse and advect, respectively, through the host polymer matrix. In the infinitely dilute limit $\phi_{1} \rightarrow 0, \tau=1$ and $\sigma=0$, and there is no hindrance to the diffusion or advection of salt. At very high polymer concentrations $\tau \rightarrow 0$ while $\sigma \rightarrow 1$, and the motion of salt is significantly hindered relative to the host matrix [15]. Given $\sigma$ and $\alpha$ the cross-diffusion factor $\ell=(\sigma+\alpha-1) / \alpha$ in $(20 c)$ can be written as

$$
\ell=1-\mathrm{e}^{-K_{12}^{\prime \prime} \phi_{1}} .
$$

The cross-diffusion factor is a measure of the viscous momentum transfer between the tracer and host particles, and is related to the Onsager phenomenological coefficients $L_{i j}$ by the relation $\ell=n_{1} L_{12} /\left(n_{2} L_{11}\right)$ [15]. Similarly to $\sigma, \ell \rightarrow 0$ as $\phi_{1} \rightarrow 0$ while $\ell \rightarrow 1$ as $\sigma \rightarrow 1$.

\subsection{Results}

With (21)-(34), the diffusion coefficients in equations (20) take the form

$$
D_{11}=D_{1}^{0}\left(1-\phi_{1}\right)^{k_{s}}\left[\frac{1+4 \phi_{1}^{e}+4 \phi_{1}^{e 2}-4 \phi_{1}^{e 3}+\phi_{1}^{e 4}}{\left(1-\phi_{1}^{e}\right)^{4}}\right]
$$




$$
\begin{gathered}
D_{12}=i D_{1}^{0}\left(1-\phi_{1}\right)^{k_{s}}\left(\mathrm{e}^{b_{12} \phi_{1}}-\mathrm{e}^{-K_{12}^{\prime \prime} \phi_{1}}\right), \\
D_{21}=\frac{\phi_{2}}{\phi_{1} \lambda^{3}}\left[\left(1-\mathrm{e}^{-K_{12}^{\prime \prime} \phi_{1}}\right) D_{11}+\phi_{1} b_{12} D_{22}\right], \\
D_{22}=D_{2}^{0} \mathrm{e}^{K_{21}^{\prime} \phi_{1}},
\end{gathered}
$$

where $\phi_{1}^{e}=\phi_{1} k_{\Pi} / 4, i=1.8$ and $b_{12}, K_{12}^{\prime \prime}$ and $K_{21}^{\prime}$ are as given in Section 4.2:

$$
b_{12}=\left(\frac{R_{1}^{e}}{R_{1}}+\lambda\right)^{3}, \quad K_{12}^{\prime \prime}=\left(\frac{k_{s}}{4.5}-\frac{k_{\eta}}{5.5}\right)\left(\frac{\lambda^{2}}{1+\lambda^{3}}-\left(1+3 \lambda+\lambda^{2}\right)\right) \quad \text { and } \quad K_{21}^{\prime}=\frac{-k_{\eta}}{1+0.16 / \lambda} \text {. }
$$

The solid blue curves in figure 1 show the $D_{i j}$ calculated from (35) using the coefficient values in Table 1 . The model shows good agreement with the data, and reproduces the nonlinear shape of the PEG cross-diffusion coefficient $D_{12}$, including the observed maximum at $\phi_{1} \approx 0.2$. A closer match to the $D_{12}$ and $D_{21}$ data can be obtained by using a best-fit value $\lambda^{\text {fit }}=0.37$ for the particle size ratio, as shown by the dotted curves in figure 1 .

\subsubsection{Molecular weight dependence}

As a further test of the model, figure 3 shows the effect on the $D_{i j}$ of increasing the polymer molecular weight. The plus symbols are data from the experiments of Vergara et al. [6] on PEG 400 and the squares are from the experiments of Annunziata et al. [5] on PEG 2000. The solid green and red curves are from equation (35) using the coefficients for PEG 400 and PEG 2000, respectively, in Table 1. The model captures the concentration dependence of $D_{11}$ and $D_{22}$ quite well for PEG 400, while somewhat over-predicting the PEG cross-diffusion coefficient $D_{12}$ and underpredicting the $\mathrm{NaCl}$ cross-diffusion coefficient $D_{21}$. Similarly to the PEG 200 case, using a smaller best-fit value for $\lambda$ leads to closer agreement with experiment.

The model predictions for PEG 2000 solutions are qualitatively similar but less accurate than for the smaller molecular weights. This may reflect a breakdown of the effective hard-sphere model - PEG 2000 molecules are much less spherical in solution than PEG 200 [32], and the PEG 2000 particles are entangled at relatively low concentrations [9]. Both of these effects negate the assumptions of the effective hard-sphere model developed here. Nevertheless, the results in figure 3 suggest the model is able to predict with a fair degree of accuracy the diffusion coefficients in low-molecular weight PEG solutions as functions of both concentration and polymer size.

\subsection{Breakdown of the Stokes-Einstein equation}

For large tracer particles with $\lambda \gg 1$, the Fickian diffusivity $D_{22}$ can be written in the form of a Stokes-Einstein equation

$$
D_{22}=\frac{k_{B} T}{6 \pi \eta R_{2}},
$$

where $\eta$ is the viscosity of the polymer solution [40,41]. Equation (36) is valid as $\lambda \rightarrow \infty$ because for large tracer particles the polymer solution behaves like an effective continuum. For small tracer particles with $\lambda<1$, however, the tracer particles are significantly affected by the molecular nature of the polymer molecules and the Stokes-Einstein relation (36) breaks down [42-44]. Physically, the tracer particles experience a reduced effective viscosity $\eta_{e}$ within the pore space that is smaller than the macroscopic viscosity $\eta[15,45,46]$. Figure 4 shows $D_{22}$ calculated from (36) with equation (15) for the PEG viscosity (dotted curves), along with experimental data for three polymer molecular weights (PEG 200, $\lambda=0.44$; PEG 400, $\lambda=0.30$; and PEG 2000, $\lambda=0.13$ ). The Stokes-Einstein relation (36) tends to underestimate the $\mathrm{NaCl}$ diffusivity, and the discrepancy becomes larger as $\lambda$ decreases.

In order to account for pore-scale viscosity effects the tracer diffusivity can be written in the form of an effective Stokes-Einstein equation

$$
D_{22}=\frac{k_{B} T}{6 \pi \eta_{e} R_{2}}
$$



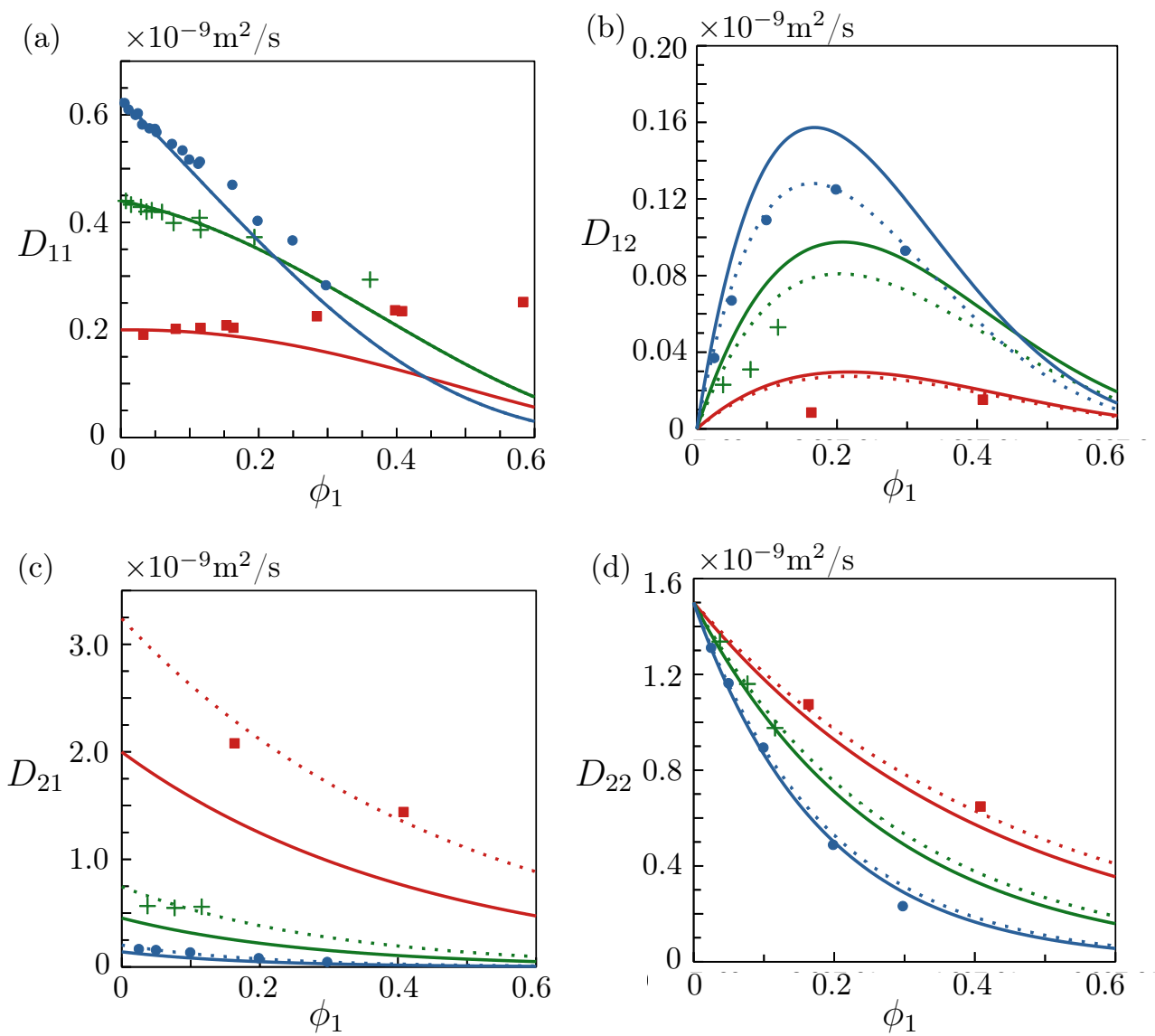

Figure 3: Cross-diffusion coefficients $D_{i j}$ in the system PEG-NaCl- $\mathrm{H}_{2} \mathrm{O}$. The data are from Vergara et al. [4] (PEG 200, 0.5 M NaCl, circles); Vergara et al. [6] (PEG 400, 0.78 M NaCl, crosses); and Annunziata et al. [5] (PEG 2000, 0.5 M NaCl, squares). Also included in (a) is mutual diffusion coefficient data from Vergara et al. [8]. The solid curves are from (35) with the coefficient values in Table 1 ; the dotted curves use the best-fit $\lambda^{\text {fit }} \approx 0.8 \lambda$ values given in Table 1 .

where $\eta_{e}$ is the effective viscosity experienced by the tracer particles within the pore space [15, 47]. Comparing (37) with (35d) gives an expression for the effective viscosity

$$
\eta_{\mathrm{e}}=\eta_{0} \exp \left[\frac{k_{\eta} \phi_{1}}{(1+0.16 / \lambda)}\right],
$$

which reduces to (15) as $\lambda \rightarrow \infty$. Equation (38) contains Batchelor's viscous correction factor $(1+0.16 / \lambda)^{-1}[3]$. The solid curves in figure 4 are from equations (37) and (38), showing much better agreement with experiment. This suggests that the breakdown of the Stokes-Einstein equation for $\mathrm{NaCl}$ diffusion in low molecular weight PEG solutions is caused mainly by long-range hydrodynamic interactions between the salt ions and the polymer matrix [48].

\subsection{Discussion}

The effective hard-sphere model developed here relies on hydrodynamic results obtained for impermeable spherical particles with no-slip boundary conditions [3]. The mobility coefficients have been modified using viscosity data leading to good results for relatively low molecular weight PEG solutions, but likely limit the model to systems in which the polymers are unentangled and roughly spherical in shape. A more general model of the hydrodynamics for polymer molecules may be porous particles with slip or partial slip boundary conditions [50, 51] or 

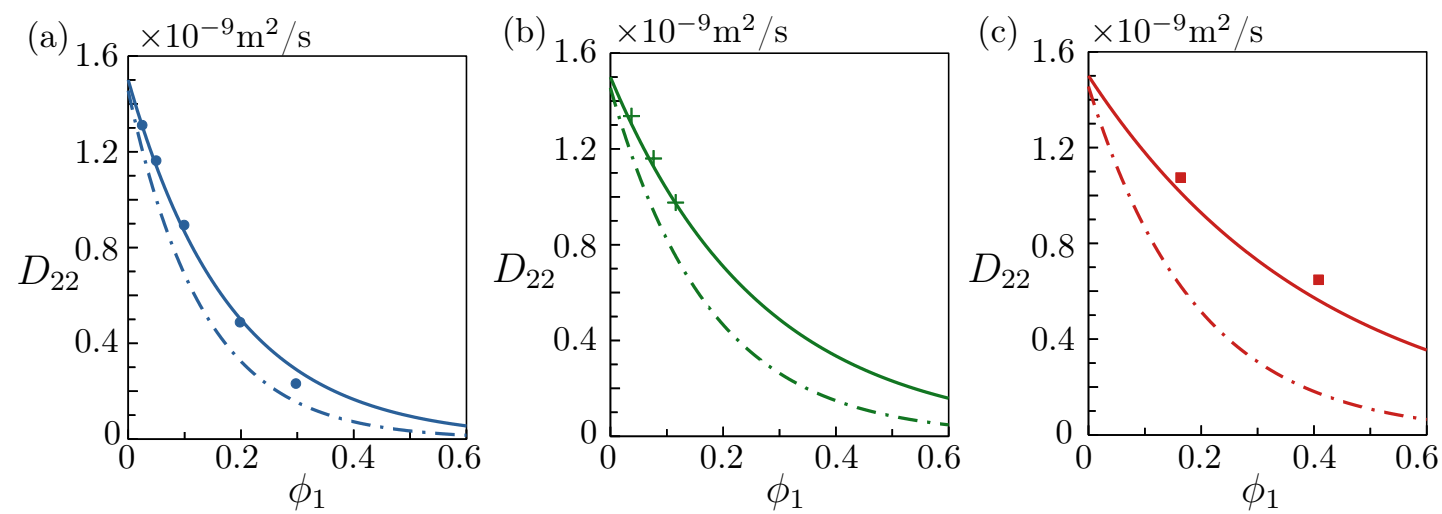

Figure 4: $\mathrm{NaCl}$ main diffusion coefficient $D_{22}$ in the system $\mathrm{PEG}-\mathrm{NaCl}-\mathrm{H}_{2} \mathrm{O}$. The data were obtained using three molecular weights: (a) PEG 200 (circles) [4]; (b) PEG 400 (crosses) [6]; and (c) PEG 2000 (squares) [49]. The dash-dot curves are from the Stokes-Einstein equation (36) and the solid curves are from equation (37).

dumbell chain models [52, 53]; however, expressions for $K_{i j}^{\prime}$ and $K_{i j}^{\prime \prime}$ for these cases are not at present available. Equations (20) may still apply even in solutions of entangled or charged macromolecules given appropriate expressions for the thermodynamic and transport coefficients. For example, Cohen et al. [54] have shown that a des Cloizeaux osmotic pressure of the form $\Pi=C_{1} R T\left[1+\alpha_{C}\left(C_{1} / C_{1}^{*}\right)^{5 / 4}\right]$ gives good agreement with experimental data in entangled PEG solutions, where $\alpha_{C}$ is a coefficient that depends on the molecular weight [55]. Similarly, the tracer diffusivity and viscosity of entangled PEG solutions can be modelled via stretched exponential functions of the form $D_{22}=D_{2}^{0} \exp \left(-\beta \phi_{1}^{\nu}\right)$ and $\eta=\eta_{0} \exp \left(a \phi_{1}^{b}\right)$, where $\beta, \nu, a$ and $b$ are constants $[44,48,56]$. An extension of the present model to entangled solutions could potentially be developed using these and similar expressions for the coefficients $\sigma, \alpha$ and $f$, and will be explored in future work.

\section{Conclusion}

An effective hard-sphere model of salt diffusion in uncharged, unentangled hydropolymer solutions has been developed. Given the viscosity, sedimentation coefficient and osmotic pressure of the polymer, the model predicts the ternary diffusion coefficients as functions of polymer size and concentration. The model uses hydrodynamic results obtained for hard spherical particles, modified using the viscosity and osmotic pressure of the polymer. This leads to good results for $\mathrm{NaCl}$ diffusion in low molecular weight polyethylene glycol solutions. It is anticipated the model will apply more generally to crowded solutions in which the host molecules are inert, unentangled and roughly spherical in shape.

\section{Acknowledgements}

This work was made possible by the love and merciful kindness of Jesus, Lord of heaven and earth and Saviour of all who believe. Believe on the Lord Jesus Christ and thou shalt be saved. Acts 16:31 


\section{A Osmotic virial coefficients}

Depending on the concentration variable used, the dilute-limit osmotic pressure of a binary solution, equation $(11 d)$, is given in the literature in several equivalent forms, including

$$
\begin{aligned}
\Pi & =n_{1} k_{B} T\left(1+B_{11} n_{1}\right) \\
& =n_{1} k_{B} T\left(1+k_{\Pi} \phi_{1}\right) \\
& =C_{1} R T\left(1+B_{11}^{*} C_{1}\right) \\
& =\rho_{1} R T\left(1 / M_{1}+A_{11} \rho_{1}\right),
\end{aligned}
$$

where $C_{1}=n_{1} / N a$ is the molar concentration $(\mathrm{mol} / \mathrm{L}), \rho_{1}=M_{1} C_{1}=M_{1} n_{1} / N a$ is the mass concentration $(\mathrm{g} / \mathrm{L}), R=k_{B} N a$ is the gas constant, $M_{1}$ is the molar mass $(\mathrm{g} / \mathrm{mol})$ and $N a$ is Avogadro's constant. Here $B_{11}$ is the second virial coefficient, $B_{11}^{*}$ is the mole-based second virial coefficient $(\mathrm{L} / \mathrm{mol})$ and $A_{11}$ is the mass based second virial coefficient $\left(\mathrm{molL} / \mathrm{g}^{2}\right)$. They are related to each other by the equations $B_{11}^{*}=B_{11} N a=k_{\Pi} v_{1} N a=M_{1}^{2} A_{11}$.

Yasukawa et al. [26] measured $A_{11}$ for several PEG molecular weights at $T=298 \mathrm{~K}$ and obtained $R T A_{11}=1.11 \times 10^{-4} \mathrm{bar} \mathrm{L} \mathrm{L}^{2} / \mathrm{g}^{2}$ for PEG 200 , where $R=0.08314 \mathrm{~L} \mathrm{bar} / \mathrm{K} / \mathrm{mol}$ is the universal gas constant, giving $A_{11}=4.48 \times 10^{-9} \mathrm{~mol} \mathrm{~m} / \mathrm{g}^{2}$ and $k_{\Pi}=M_{1}^{2} A_{11} /\left(\mathrm{Nav}_{1}\right)=1.81$, where $M_{1}=200 \mathrm{~g} / \mathrm{mol}$. Gaube et al. [57] also measured $A_{11}$ for PEG 200, at a slightly lower temperature of $293 \mathrm{~K}$, and obtained a larger value $A_{11}=7.16 \times 10^{-9} \mathrm{~mol} \mathrm{~m}^{3} / \mathrm{g}^{2}$, giving $k_{\Pi}=2.92$. Here the data of Yasukawa et al. [26] is used, as the experiments were conducted at the same temperature as the PEG cross-diffusion experiments [4].

For PEG 600 Yasukawa et al. [26] obtained $R T A_{11}=5.86 \times 10^{-5} \mathrm{bar} \mathrm{L}^{2} / \mathrm{g}^{2}$; extrapolation between the PEG 200 and PEG 600 values gives $R T A_{11} \approx 8.48 \times 10^{-5} \mathrm{bar} \mathrm{L}^{2} / \mathrm{g}^{2}$ for PEG 400, yielding $k_{\Pi}=1.85$. Finally, for PEG $2000 R T A_{11}=4.00 \times 10^{-5} \mathrm{bar} \mathrm{L}^{2} / \mathrm{g}^{2}[26]$ giving $k_{\Pi}=1.98$.

\section{References}

[1] E. L. Cussler. Diffusion: Mass Transfer in Fluid Systems. Cambridge University Press, U.K., 2009.

[2] J. S. Kirkaldy. Diffusion in multicomponent metallic systems. Canadian Journal of Physics, 35, 435-440, 1957.

[3] G. K. Batchelor. Diffusion in a dilute polydisperse system of interacting spheres. Journal of Fluid Mechanics, 131, 155-175, 1983; and Corrigendum 137, 467-469, 1983.

[4] A. Vergara, O. Annunziata, L. Paduano, D. G. Miller, J. G. Albright, and R. Sartorio. Multicomponent Diffusion in Crowded Solutions. 2. Mutual Diffusion in the Ternary System tetra(ethylene glycol)-NaCl-water. The Journal of Physical Chemistry B, 108, 2764-2772, 2004.

[5] O. Annunziata, A. Vergara, L. Paduano, R. Sartorio, D. G. Miller, and J. G. Albright. Quaternary diffusion coefficients in a protein-polymer-salt-water system determined by Rayleigh interferometry. The Journal of Physical Chemistry B, 113, 13446-13453, 2009.

[6] A. Vergara, L. Paduano, V. Vitagliano, and R. Sartorio. Multicomponent diffusion in crowded solutions. 1. Mutual diffusion in the ternary system poly(ethylene glycol) $400-\mathrm{NaCl}-$ water. Macromolecules, 34, 991-1000, 2001.

[7] O. Annunziata, D. Buzatu, and J. G. Albright. Protein diffusiophoresis and salt osmotic diffusion in aqueous solutions. The Journal of Physical Chemistry B, 116, 12694-12705, 2012.

[8] A. Vergara, L. Paduano, V. Vitagliano, and R. Sartorio. Mutual diffusion in aqueous solution of poly(ethylene glycol) samples. Some comments on the effect of chain length and polydispersity. Phys. Chem. Chem. Phys., 1, 5377-5383, 1999. 
[9] A. Vergara, L. Paduano, G. D'Errico, and R. Sartorio. Network formation in polyethylene glycol solutions. An intradiffusion study. Phys. Chem. Chem. Phys., 1, 4875-4879, 1999.

[10] V. Vitagliano and P. A. Lyons. Diffusion coefficients for aqueous solutions of sodium chloride and barium chloride. Journal of the American Chemical Society, 78, 1549-1552, 1956.

[11] S. R. deGroot and P. Mazur. Non-Equilibrium Thermodynamics. North-Holland Publishing Co., Amsterdam, 1962.

[12] O. Annunziata. On the role of solute solvation and excluded-volume interactions in coupled diffusion. The Journal of Physical Chemistry B, 112, 11968-11975, 2008.

[13] G. K. Batchelor. Note on the Onsager symmetry of the kinetic coefficients for sedimentation and diffusion in a dilute bidispersion. Journal of Fluid Mechanics, 171, 509-517, 1986.

[14] A. Santos and R. D. Rorhmann. Chemical-potential route for multicomponent fluids. Phys. Rev. E, 87, 052138, 2013.

[15] S. S. L. Peppin. Theory of tracer diffusion in concentrated hard-sphere suspensions. J. Fluid Mech., 870, 1105-1126, 2019.

[16] G. K. Batchelor. Brownian diffusion of particles with hydrodynamic interaction. Journal of Fluid Mechanics, 74, 1-29, 1976.

[17] W. B. Russel, D. A. Seville, and W. R. Schowalter. Colloidal Dispersions. Cambridge University Press, U.K., 1989.

[18] A. P. Minton. The effective hard particle model provides a simple, robust, and broadly applicable description of nonideal behavior in concentrated solutions of bovine serum albumin and other nonassociating proteins. Journal of Pharmaceutical Sciences, 96, 3466-3469, 2007.

[19] Zhe Wang, Antonio Faraone, Panchao Yin, Lionel Porcar, Yun Liu, Changwoo Do, Kunlun Hong, and Wei-Ren Chen. Dynamic equivalence between soft star polymers and hard spheres. ACS Macro Letters, 8, 1467-1473, 2019.

[20] A. J. Rowe. The concentration dependence of transport processes: A general description applicable to the sedimentation, translational diffusion, and viscosity coefficients of macromolecular solutes. Biopolymers, 16, 2595-2611, 1977.

[21] M. M. Kops-Werkhoven and H. M. Fijnaut. Dynamic light scattering and sedimentation experiments on silica dispersions at finite concentrations. The Journal of Chemical Physics, 74, 1618-1625, 1981.

[22] M. Wales and K. E. Van Holde. The concentration dependence of the sedimentation constants of flexible macromolecules. Journal of Polymer Science, 14, 81-86, 1954.

[23] J. G. Albright, L. Paduano, R. Sartorio, A. Vergara, and V. Vitagliano. Multicomponent diffusion in systems containing molecules of different size. 1. Mutual diffusion in the ternary system poly(ethylene glycol) $2000+$ poly(ethylene glycol) $200+$ water. Journal of Chemical E Engineering Data, 46, 1283-1291, 2001.

[24] D. K. Thomas and T. A. J. Thomas. Viscosity - concentration relationships in solutions of high polymers. Journal of Applied Polymer Science, 3, 129-131, 1960.

[25] George D. J. Phillies. The hydrodynamic scaling model for polymer self-diffusion. The Journal of Physical Chemistry, 93, 5029-5039, 1989.

[26] Masahiro Yasukawa, Yasuhiro Tanaka, Tomoki Takahashi, Masafumi Shibuya, Shoji Mishima, and Hideto Matsuyama. Effect of molecular weight of draw solute on water permeation in forward osmosis process. Industrial $\&$ Engineering Chemistry Research, 54, 8239-8246, 2015. 
[27] John J. Correia and Walter F. Stafford. Sedimentation velocity: A classical perspective. In James L. Cole, editor, Analytical Ultracentrifugation, volume 562 of Methods in Enzymology, pages 49 - 80. Academic Press, 2015.

[28] J.M. Creeth and C.G. Knight. On the estimation of the shape of macromolecules from sedimentation and viscosity measurements. Biochimica et Biophysica Acta (BBA) - Biophysics including Photosynthesis, 102, 549 - 558, 1965.

[29] Hwankyu Lee, Richard M. Venable, Alexander D. MacKerell, and Richard W. Pastor. Molecular dynamics studies of polyethylene oxide and polyethylene glycol: Hydrodynamic radius and shape anisotropy. Biophysical Journal, 95, 1590 - 1599, 2008.

[30] M. Muthukumar. Concentration dependent friction coefficients of polymer molecules in dilute solutions. I. The Journal of Chemical Physics, 78, 2764-2772, 1983.

[31] R. Heyrovská. Physical electrochemistry of strong electrolytes based on partial dissociation and hydration: Quantitative interpretation of the thermodynamic properties of $\mathrm{NaCl}(\mathrm{aq})$ from "zero to saturation". Journal of The Electrochemical Society, 143, 1789-1793, 1996.

[32] D. K. Thomas and A. Charlesby. Viscosity relationship in solutions of polyethylene glycols. Journal of Polymer Science, 42, 195-202, 1960.

[33] Michele S. McAfee, Huixiang Zhang, and Onofrio Annunziata. Amplification of salt-induced polymer diffusiophoresis by increasing salting-out strength. Langmuir, 30, 12210-12219, 2014.

[34] R. J. Phillips. Gradient diffusion in dilute suspensions of hard spheroidal particles. Journal of Fluid Mechanics, 887, A1-1-34, 2020.

[35] R. J. Ellis and A. P. Minton. Join the crowd. Nature, 425, 27-28, 2003.

[36] M. S. McAfee and O. Annunziata. Effect of particle size on salt-induced diffusiophoresis compared to Brownian mobility. Langmuir, 30, 4916-4923, 2014.

[37] S. S. L. Peppin, J. A. W. Elliott, and M. G. Worster. Pressure and relative motion in colloidal suspensions. Physics of Fluids, 17, 053301, 2005.

[38] J. F. Richardson and W. N. Zaki. Sedimentation and fluidisation. part 1. Trans. Inst. Chem. Eng., 32, 35-53, 1954.

[39] C. D. Shackelford. Laboratory testing for waste disposal - A review. J. Contam. Hydrol., 7, 177-217, 1991.

[40] A. Einstein. Investigations on the theory of Brownian movement. Dover, U.S.A., 1956.

[41] W. Sutherland. A dynamical theory of diffusion for non-electrolytes and the molecular mass of albumin. The London, Edinburgh, and Dublin Philosophical Magazine and Journal of Science, 9, 781-785, 1905.

[42] Anish Tuteja, Michael E. Mackay, Suresh Narayanan, Subashini Asokan, and Michael S. Wong. Breakdown of the continuum Stokes-Einstein relation for nanoparticle diffusion. Nano Letters, 7, 1276-1281, 2007.

[43] Jun Liu, Dapeng Cao, and Liqun Zhang. Molecular dynamics study on nanoparticle diffusion in polymer melts: A test of the Stokes-Einstein law. The Journal of Physical Chemistry C, 112, 6653-6661, 2008.

[44] R. Holyst, A. Bielejewska, J. Szymański, A. Wilk, A. Patkowski, J. Gapiński, A. Żywociński, T. Kalwarczyk, E. Kalwarczyk, M. Tabaka, N. Ziębacz, and S. A. Wieczorek. Scaling form of viscosity at all length-scales in poly(ethylene glycol) solutions studied by fluorescence correlation spectroscopy and capillary electrophoresis. Phys. Chem. Chem. Phys., 11, 9025-9032, 2009. 
[45] Tai-Hsi Fan, Jan K. G. Dhont, and Remco Tuinier. Motion of a sphere through a polymer solution. Phys. Rev. E, 75, 011803, 2007.

[46] Karol Makuch, Robert Holyst, Tomasz Kalwarczyk, Piotr Garstecki, and John F. Brady. Diffusion and flow in complex liquids. Soft Matter, 16, 114-124, 2020.

[47] T. Kalwarczyk, K. Sozanski, S. Jakiela, A. Wisniewska, E. Kalwarczyk, K. Kryszczuk, S. Hou, and R. Holyst. Length-scale dependent transport properties of colloidal and protein solutions for prediction of crystal nucleation rates. Nanoscale, 6, 10340-10346, 2014.

[48] Gregory S. Ullmann, Kathleen Ullmann, Robert M. Lindner, and George D. J. Phillies. Probe diffusion of polystyrene latex spheres in polyethylene oxide-water. The Journal of Physical Chemistry, 89, 692-700, 1985.

[49] H. Zhang and O. Annunziata. Macromolecular hydration compared with preferential hydration and their role on macromolecule-osmolyte coupled diffusion. Phys. Chem. Chem. Phys., 11, 8923-8932, 2009.

[50] R.B. Jones. Diffusion of tagged interacting spherically symmetric polymers. Physica A: Statistical Mechanics and its Applications, 97, 113 - 126, 1979.

[51] Wei C. Lin and Huan J. Keh. Diffusiophoresis in suspensions of charged soft particles. Colloids and Interfaces, 4(3), 1-16, 2020.

[52] Hiromi Yamakawa. Transport properties of polymer chains in dilute solution: Hydrodynamic interaction. The Journal of Chemical Physics, 53, 436-443, 1970.

[53] James W. Swan and Gang Wang. Rapid calculation of hydrodynamic and transport properties in concentrated solutions of colloidal particles and macromolecules. Physics of Fluids, 28, 011902, 2016.

[54] J. A. Cohen, R. Podgornik, P. L. Hansen, and V. A. Parsegian. A phenomenological oneparameter equation of state for osmotic pressures of PEG and other neutral flexible polymers in good solvents. The Journal of Physical Chemistry B, 113, 3709-3714, 2009.

[55] J. Li, M. Turesson, C. A. Haglund, B. Cabane, and M. Skepo. Equation of state of PEG/PEO in good solvent. Comparison between a one-parameter EOS and experiments. Polymer, 80, $205-213,2015$.

[56] Indermeet Kohli and Ashis Mukhopadhyay. Diffusion of nanoparticles in semidilute polymer solutions: Effect of different length scales. Macromolecules, 45, 6143-6149, 2012.

[57] Johann Gaube, Andreas Pfennig, and Matthias Stumpf. Vapor-liquid equilibrium in binary and ternary aqueous solutions of poly(ethylene glycol) and dextran. Journal of Chemical $\mathbb{6}$ Engineering Data, 38, 163-166, 1993. 